\title{
Superimposed Training-Based Channel Estimation for MIMO Relay Networks
}

\author{
Xiaoyan Xu, Jianjun Wu, Shubo Ren, Lingyang Song, and Haige Xiang \\ School of Electronics Engineering and Computer Science, Peking University, Beijing 100871, China \\ Correspondence should be addressed to Jianjun Wu, just@pku.edu.cn \\ Received 31 May 2012; Accepted 14 July 2012 \\ Academic Editor: Sumei Sun \\ Copyright () 2012 Xiaoyan Xu et al. This is an open access article distributed under the Creative Commons Attribution License, \\ which permits unrestricted use, distribution, and reproduction in any medium, provided the original work is properly cited.
}

We introduce the superimposed training strategy into the multiple-input multiple-output (MIMO) amplify-and-forward (AF) one-way relay network (OWRN) to perform the individual channel estimation at the destination. Through the superposition of a group of additional training vectors at the relay subject to power allocation, the separated estimates of the source-relay and relay-destination channels can be obtained directly at the destination, and the accordance with the two-hop AF strategy can be guaranteed at the same time. The closed-form Bayesian Cramér-Rao lower bound (CRLB) is derived for the estimation of two sets of flat-fading MIMO channel under random channel parameters and further exploited to design the optimal training vectors. A specific suboptimal channel estimation algorithm is applied in the MIMO AF OWRN using the optimal training sequences, and the normalized mean square error performance for the estimation is provided to verify the Bayesian CRLB results.

\section{Introduction}

In recent years, the use of relays has gained significant interest for the advantage of enhancing link reliability and increasing channel capacity in a wireless network. By transmitting the signal through one or more relays located at geographically separated nodes, the effect of signal fading due to multipath propagation and strong shadowing can be compensated through the exploitation of spatial diversity provided by the network nodes, as well as the efficient use of power resources, which can be achieved by a scheme that simply receives and forwards a given information, yet designed under certain optimality criterion. In particular, the source can cooperate with the relay to forward the replicas of the source signal to the destination thereby providing additional diversity and improved signal quality at the destination. There has been several relaying strategies, of which the most dominant are amplify-and-forward (AF) and decode-and-forward (DF).

Multiple-input multiple-output (MIMO) technology, by providing significant improvements in terms of spectral efficiency and link reliability, has been introduced to achieve high data rates required by the next-generation wireless communication systems. Since MIMO systems are able to support high-data rates by combating fading and interference, coupling relay networks with MIMO techniques is a natural extension to the state of the art. The basic idea is to introduce relays that forward the data to the destination, which is otherwise out of the reach of the source. With twohop relaying, we can increase the rank and consequently the capacity of ill-conditioned (rank deficient) MIMO channels. Relaying information on two-hops also decreases the need for high power at the transmitter. For MIMO relay channels where every terminal in the wireless network can be deployed with multiple antennas, studies are mainly concentrated on spatial multiplexing (SM) systems. The potentiality of MIMO relay channels in wireless networks has been discussed and several results on capacity bounds have been found in previous works [1-3], and the optimal relay beamforming design and the corresponding transceivers design have been studied in MIMO scenarios [4-8].

Before enjoying all the benefits brought by the MIMO relay network, an accurate channel state information (CSI) is required at the destination (for AF) or at both relay and destination (for DF). However, most of the existing works 
are based on the assumption that all nodes have perfect CSI, which is actually the perfect knowledge of backward (sourcerelay) and forward (relay-destination) channels. Recently, the estimation algorithms for the cascaded channel of sourcerelay-destination link have been proposed in [9-12] for AF-based MIMO relay systems, as data detection at the destination only requires the knowledge of the cascaded channel.

However, only the cascaded channel information is not enough in some other applications, and the individual information of both the backward and forward channels is needed for the destination to perform certain operations. For example, in relay beamforming schemes [13, 14], especially in noncoherent MIMO relay networks, where the optimization process is performed at the destination [7], the individual CSIs are utilized to design the optimal amplifying matrix at the relay and the power allocation between the source and relay so as to maximize the instantaneous capacity of the two-hop MIMO AF relay system. Another application is the subcarrier pairing, which is performed at the relay to allocate power over subcarriers on the two-hops such that the instantaneous rate of the relay link is maximized $[15,16]$, the destination also needs the individual CSIs in order to know the pairing strategy used at the relay.

Conventionally, the backward and forward channels can be estimated directly at the relay and destination, respectively, and then the relay spends additional time slots to send the backward channel estimation to the destination through the feedback channel. Apart from the additional energy and time slots consumed on the feedback transmission, the feedback information are subject to further distortion such as the quantization errors of the channel estimates and the errors in the communication through the feedback channel. An alternate way to estimate the individual channels at the destination is to apply a three-phase training strategy, that is, after the two phase training as in the estimation of the cascaded channel, the relay sends its own training to the destination so that the individual channels can be estimated $[17,18]$. However, a critical drawback of this strategy is its incompatibility with the two-phase data transmission. Moreover, as demonstrated in $[14,19,20]$, the performance of a two-hop MIMO AF relay system can be improved if the individual CSIs are exploited.

Therefore, in a practical scenario, it is important to design a MIMO relay scheme that provides both in accordance with the two-hop data transmission and the estimates of individual channels at the destination. In [21], a least square (LS) method is developed to estimate the individual channels from the singular value decomposition (SVD) of the cascaded channel.

Inspired by the superimposed training in point-to-point communications, $[22,23]$ designed a superimposed training strategy in AF OWRN, where all the nodes are equipped with single antenna. We extend the research to multiantenna scenario and introduce the superimposed training strategy into MIMO AF OWRN in this work. The individual channel estimation is accomplished at the destination, and the closed-form Bayesian Cramér-Rao lower bound (CRLB) is derived for the estimation of flat-fading MIMO channels,

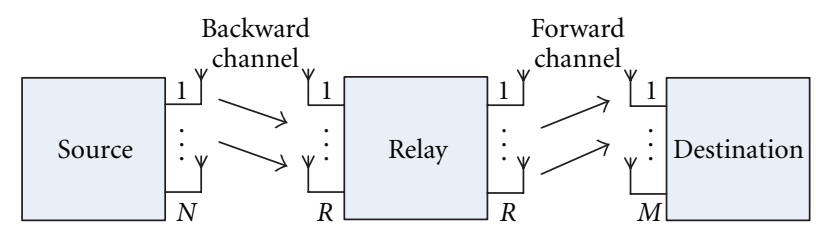

Figure 1: A MIMO relay system.

which is further used to guide the optimal training design. Specifically, the relay superimposes its own training signal over the received signal before forwarding it out, which provides the separated channel information, and the total relay power is allocated between the two parts reasonably. The simulation is provided to verify the Bayesian CRLB results by the normalized mean square error (NMSE) performance of a specific suboptimal channel estimation algorithm. The extracted individual channel information can be used to help predict the actions of the relay for data transmission, such as power allocation and carrier permutation, without the need of feedback from the relay.

The rest of the paper is organized as follows. The system model of superimposed training in the MIMO AF OWRN is given in Section 2. In Section 3, the Bayesian CRLB of superimposed training-based channel estimation is derived and used to design the optimal training sequences. A suboptimal channel estimation algorithm is described in Section 4. Finally, the simulation results are provided in Section 5 and conclusions are drawn in Section 6.

Notations. Vectors and matrices are boldface small and capital letters. The Hermitian, inverse, and pseudoinverse of the matrix $\mathbf{A}$ are denoted by $\mathbf{A}^{H}, \mathbf{A}^{-1}$, and $\mathbf{A}^{\dagger}$, respectively. $\operatorname{tr}(\mathbf{A})$ and $\|\mathbf{A}\|_{F}$ are the trace and the Frobenius norm of $\mathbf{A}$. $[\mathbf{A}]_{i j}$ is the $(i, j)$ th entry of $\mathbf{A}$, and $\operatorname{diag}\{\mathbf{a}\}$ denotes a diagonal matrix with the diagonal element constructed from $\mathbf{a}$. I is the identity matrix, and $\mathbf{e}_{i}$ is the basis vector with the $i$ th element 1 and 0 otherwise. $E\{\cdot\}$ denotes the statistical expectation, and $\otimes$ denotes the Kronecker product.

\section{System Model}

Consider a nonregenerative MIMO relay system as in Figure 1, there is one source with $N$ antennas, one destination with $M$ antennas, and one relay with $R$ receive antennas and $R$ transmit antennas. It is assumed that there is no spatial correlation at the relay, and the direct link between the source and destination is ignored due to the larger distance and additional pathloss compared to the relay link. When SM is applied at the source, it requires $M \geq N$ and $R \geq N$ such that the compound channel can support $N$ independent substreams. For expositional purpose, we assume without loss of generality $M \leq R$ in the following. However, our results below can be extended easily to the case $M>R$. Through this paper, we assume perfect synchronization among all terminals, and the total transmit power for the source and the relay is the same.

We restrict our discussion to the case of a slow, frequency-flat block fading model. The data transmission 
takes place in two time slots using two-hops. During the first time slot, the source transmits the signal to the relay and yields the received signal as

$$
\mathbf{r}(n)=\mathbf{H}_{1} \mathbf{x}(n)+\mathbf{w}_{R}(n),
$$

where $\mathbf{r}(n)$ is the $R \times 1$ received signal vector at the relay, $\mathbf{x}(n)$ is the $N \times 1$ transmit signal vector with covariance matrix $\left(\rho_{s} / N\right) \mathbf{I}_{N}$, and $\rho_{s}$ denotes the transmit power of the source. $\mathbf{H}_{1} \in \mathbb{C}^{R \times N}$ denotes the backward channel matrix and its entries are assumed to be zero-mean circular symmetric complex Gaussian (ZMCSCG) random variables of unit variance. $\mathbf{w}_{R}(n)$ is the $R \times 1$ complex circular additive white Gaussian noise (AWGN) vector at the relay with zero mean and covariance matrix $\sigma_{n}^{2} \mathbf{I}_{R}$.

The relay processes the received signal and superimposes a new training vector $\mathbf{t}_{r}$ over it. The forwarded signal at the relay can be written as

$$
\mathbf{r}_{t}(n)=\alpha \mathbf{G}\left[\mathbf{H}_{1} \mathbf{x}(n)+\mathbf{w}_{R}(n)\right]+\mathbf{t}_{r}(n),
$$

where the scalar $\alpha$ controls the power allocation between the received signal and the superimposed training, $\mathbf{G}=$ $\sqrt{\left(\rho_{r} / R\right) /\left(1+\rho_{s}\right)} \mathbf{I}_{R}$ is a suboptimal but convenient forwarding scaling matrix with $\sigma_{n}^{2}$ fixed as 1 , and $\rho_{r}$ corresponds to the transmit power of the relay. The following constraint should be satisfied for the training block:

$$
E\left\{\left\|\mathbf{r}_{t}(n)\right\|_{F}^{2}\right\}=\alpha^{2} \frac{\left(\rho_{r} / R\right)}{1+\rho_{s}}\left(R \rho_{s}+R \sigma_{n}^{2}\right)+\mathbf{t}_{r}^{H} \mathbf{t}_{r} \leq \rho_{r} .
$$

For the power allocation scheme at the relay, define $\rho_{t}=$ $\mathbf{t}_{r}^{H}(n) \mathbf{t}_{r}(n)$ as the average power assigned for the superimposed training at the relay, and then we have

$$
\rho_{t}=\rho_{r}-\alpha^{2} \frac{\left(\rho_{r} / R\right)}{1+\rho_{s}}\left(R \rho_{s}+R \sigma_{n}^{2}\right)=\rho_{r}\left(1-\alpha^{2}\right) .
$$

The received signal at the destination is represented as

$$
\begin{aligned}
\mathbf{y}(n) & =\alpha \mathbf{H}_{2} \mathbf{G r}(n)+\mathbf{H}_{2} \mathbf{t}_{r}(n)+\mathbf{w}_{D}(n) \\
& =\alpha \mathbf{H}_{2} \mathbf{G} \mathbf{H}_{1} \mathbf{x}(n)+\mathbf{H}_{2} \mathbf{t}_{r}(n)+\alpha \mathbf{H}_{2} \mathbf{G w}_{R}(n)+\mathbf{w}_{D}(n) \\
& \triangleq \mathbf{H} \mathbf{x}(n)+\mathbf{H}_{2} \mathbf{t}_{r}(n)+\mathbf{w}(n),
\end{aligned}
$$

where $\mathbf{H}_{2} \in \mathbb{C}^{M \times R}$ denotes the forward channel matrix, and its entries are assumed to be ZMCSCG random variables of unit variance. $\mathbf{w}_{D}(n)$ is the $M \times 1$ complex circular AWGN vector at the destination with zero mean and covariance matrix $\sigma_{n}^{2} \mathbf{I}_{M} . \mathbf{H}=\alpha \mathbf{H}_{2} \mathbf{G H}_{1}$ is defined as the cascaded channel from the source to the destination, and $\mathbf{w}(n)=$ $\alpha \mathbf{H}_{2} \mathbf{G w}_{R}(n)+\mathbf{w}_{D}(n)$ is the overall noise at the destination.

For channel estimation purposes, we assume that two sets of known training sequences $\mathbf{X}=\left[\mathbf{x}_{1}, \ldots, \mathbf{x}_{L}\right]$ and $\mathbf{T}_{r}=\left[\mathbf{t}_{r 1}, \ldots, \mathbf{t}_{r L}\right]$ of length $L$ is transmitted, where $L \geq N$. The optimal training sequences are orthogonal across all transmitting antennas [23], that is,

$$
\mathbf{X X}^{H}=L \mathbf{I}_{N}, \quad \mathbf{T}_{r} \mathbf{T}_{r}^{H}=L \mathbf{I}_{R} .
$$

Then the data model during one training period can be expressed as

$$
\mathbf{Y}=\alpha \mathbf{H}_{2} \mathbf{G H}_{1} \mathbf{X}+\mathbf{H}_{2} \mathbf{T}_{r}+\mathbf{N}
$$

where $\mathbf{N}=[\mathbf{w}(1), \ldots, \mathbf{w}(L)]$ is the compound noise matrix during the ith training block. The task of channel estimation in the MIMO OWRN is to find $\mathbf{H}_{1}$ and $\mathbf{H}_{2}$ from $\mathbf{Y}$ at the destination.

\section{Bayesian Cramér-Rao Bound and the Train- ing Design}

For many practical estimation problems, popular estimators such as the maximum likelihood (ML) estimator or the maximum a posteriori (MAP) estimator are infeasible, so one has to resort to suboptimal estimators, which are typically evaluated by determining mean square error (MSE) through simulations and by comparing this error to theoretical performance bounds. In particular, the family of CramérRao bound (CRB) has been shown to give tight estimation lower bounds in a number of practical scenarios.

3.1. Bayesian Cramér-Rao Bound. The CRB for the estimation of deterministic parameters is given by the inverse of the FIM, and Van Trees derived an analogous bound to the $\mathrm{CRB}$ for random variables, referred to as "Bayesian CRB" (BCRB). Unlike the standard and modified CRBs, the statistical dependence is naturally considered within the BCRB framework. With the assist of BCRB, the performance of the suboptimal estimators in the MIMO OWRN can be assessed, and the optimal training design can be obtained. Given the parameter vector $\theta=\left[\mathbf{h}_{1}^{T}, \mathbf{h}_{2}^{T}\right]^{T}$, the Gaussian random vector to be estimated, where $\mathbf{h}_{1}=\operatorname{vec}\left(\mathbf{H}_{1}\right)$ and $\mathbf{h}_{2}=\operatorname{vec}\left(\mathbf{H}_{2}\right)$ are the vector forms of $\mathbf{H}_{1}$ and $\mathbf{H}_{2}$, and the Bayesian FIM is defined as

$$
\mathscr{g}=E\left\{\frac{\partial \ln p(\mathbf{Y}, \theta)}{\partial \theta^{*}}\left(\frac{\partial \ln p(\mathbf{Y}, \theta)}{\partial \theta^{*}}\right)^{H}\right\}=\left[\begin{array}{ll}
\mathscr{g}_{11} & \mathscr{g}_{12} \\
\mathscr{g}_{12}^{H} & \mathscr{g}_{22}
\end{array}\right],
$$

where the expectation is taken over the joint probability density function (pdf) $p(\mathbf{Y}, \theta)$.

Lemma 1. The Bayesian FIM is computed as

$$
\begin{gathered}
g_{11}=c_{1}\left(\mathbf{X}^{*} \mathbf{X}^{T}\right) \otimes \mathbf{I}_{R}+\mathbf{R}_{\mathbf{h}_{1}}^{-1}, \\
g_{12}=\mathbf{0}_{N R \times M R}, \\
g_{21}=\mathbf{0}_{M R \times N R}, \\
g_{22}=c_{2} \operatorname{tr}\left(\mathbf{X}^{*} \mathbf{X}^{T}\right) \mathbf{I}_{M R}+c_{3}\left(\mathbf{T}_{r}^{*} \otimes \mathbf{I}_{M}\right)\left(\mathbf{T}_{r}^{T} \otimes \mathbf{I}_{M}\right) \\
+c_{4} \mathbf{I}_{M R}+\mathbf{R}_{\mathbf{h}_{2}}^{-1},
\end{gathered}
$$


where $\mathbf{R}_{\mathbf{h}_{1}}$ and $\mathbf{R}_{\mathbf{h}_{2}}$ are the covariance matrices of $\mathbf{h}_{1}$ and $\mathbf{h}_{2}$, respectively, and

$$
\begin{gathered}
c_{1}=\frac{\alpha^{2} \rho_{r}}{\sigma_{n}^{2} R\left(1+\rho_{s}+\alpha^{2} \rho_{r}\right)}, \\
c_{2}=\frac{\alpha^{2} \rho_{r}}{\sigma_{n}^{2} R\left(1+\rho_{s}+\alpha^{2} \rho_{r}\right)}, \\
c_{3}=\frac{\left(1+\rho_{s}\right)}{\sigma_{n}^{2}\left(1+\rho_{s}+\alpha^{2} \rho_{r}\right)}, \\
c_{4}=\frac{\alpha^{4} L M \rho_{r}^{2}\left[\left(1+\rho_{s}\right)+\sigma_{n}^{2}\left(1+\rho_{s}+\alpha^{2} \rho_{r}\right)\right]}{R^{2}\left(1+\rho_{s}\right)\left(1+\rho_{s}+\alpha^{2} \rho_{r}\right)^{2}} .
\end{gathered}
$$

Proof. See Appendix A.

Before proceeding, we give the Bayesian FIM results for two special cases: $\alpha=0$ and $\alpha=1$. Fix the parameters $N=$ $R=M, \sigma_{n}^{2}=1, \rho_{s}=\rho_{r}$, and $\alpha=0$ corresponds to the case when all the relay power is allocated to the superimposed training, then we have

$$
\begin{gathered}
g_{11}=\mathbf{R}_{\mathbf{h}_{1}}^{-1}, \\
g_{12}=\mathbf{0}_{N R \times M R}, \\
g_{21}=\mathbf{0}_{M R \times N R}, \\
g_{22}=\left(\mathbf{T}_{r}^{*} \otimes \mathbf{I}_{M}\right)\left(\mathbf{T}_{r}^{T} \otimes \mathbf{I}_{M}\right)+\mathbf{R}_{\mathbf{h}_{2}}^{-1} .
\end{gathered}
$$

The results for $\alpha=0$ are in line with our expectation, since the superimposed training occupies all the relay power, which means the original training from the source is discarded by the relay, the direct outcome is that the training power only works on the estimation of $\mathbf{H}_{2}$.

As for $\alpha=1$, all the relay power is used to amplify and forward the received signal from the source, and $\rho_{t}=0$, then we have

$$
\begin{gathered}
\mathscr{g}_{11}=c_{1}\left(\mathbf{X}^{*} \mathbf{X}^{T}\right) \otimes \mathbf{I}_{R}+\mathbf{R}_{\mathbf{h}_{1}}^{-1}, \\
\mathscr{g}_{12}=\mathbf{0}_{N R \times M R}, \\
\mathscr{g}_{21}=\mathbf{0}_{M R \times N R}, \\
g_{22}=c_{2} \operatorname{tr}\left(\mathbf{X}^{*} \mathbf{X}^{T}\right) \mathbf{I}_{M R}+c_{4} \mathbf{I}_{M R}+\mathbf{R}_{\mathbf{h}_{2}}^{-1},
\end{gathered}
$$

where

$$
\begin{gathered}
c_{1}=\frac{\rho_{s}}{R\left(1+2 \rho_{s}\right)}, \\
c_{2}=\frac{\rho_{s}}{R\left(1+2 \rho_{s}\right)}, \\
c_{4}=\frac{L \rho_{s}^{2}\left(2+3 \rho_{s}\right)}{R\left(1+\rho_{s}\right)\left(1+2 \rho_{s}\right)^{2}} .
\end{gathered}
$$

The results for $\alpha=1$ are also expectable since the training power is concentrated in the original training sequences from the source, which works on the estimation of both $\mathbf{H}_{1}$ and $\mathbf{H}_{2}$ at the same time.
Since $\boldsymbol{g}_{12}=\mathbf{0}_{N R \times M R}$ and $\boldsymbol{g}_{21}=\mathbf{0}_{M R \times N R}$, the BCRBs for $\mathbf{H}_{1}$ and $\mathbf{H}_{2}$ can be separately expressed as

$$
\mathrm{BCRB}_{\mathbf{h}_{1}}=g_{11}^{-1}, \quad \mathrm{BCRB}_{\mathbf{h}_{2}}=g_{22}^{-1},
$$

and the channel error covariances are lower bounded by

$$
\begin{aligned}
& \operatorname{Cov}_{\mathbf{h}_{1}}=\mathrm{E}\left\{\Delta \mathbf{h}_{1} \Delta \mathbf{h}_{1}^{H}\right\} \succeq \mathrm{BCRB}_{\mathbf{h}_{1}}, \\
& \operatorname{Cov}_{\mathbf{h}_{2}}=\mathrm{E}\left\{\Delta \mathbf{h}_{2} \Delta \mathbf{h}_{2}^{H}\right\} \succeq \mathrm{BCRB}_{\mathbf{h}_{2}},
\end{aligned}
$$

where $\mathbf{X} \succeq \mathbf{Y}$ means that $\mathbf{X}-\mathbf{Y}$ is positive semidefinite. Furthermore, the channel estimation MSEs are lower bounded by

$$
\begin{aligned}
& \operatorname{MSE}_{\mathbf{h}_{1}}=\operatorname{tr}\left(\operatorname{Cov}_{\mathbf{h}_{1}}\right) \geq \operatorname{tr}\left(\mathrm{BCRB}_{\mathbf{h}_{1}}\right), \\
& \operatorname{MSE}_{\mathbf{h}_{2}}=\operatorname{tr}\left(\operatorname{Cov}_{\mathbf{h}_{2}}\right) \geq \operatorname{tr}\left(\mathrm{BCRB}_{\mathbf{h}_{2}}\right) .
\end{aligned}
$$

3.2. Training Design from Bayesian Cramér-Rao Lower Bound. For the purpose of minimizing the MSEs in the channel estimation, the two optimizations on the training sequences in the MIMO AF OWRN are formulated as

$$
\begin{array}{ll}
\min _{\mathbf{X}} & \operatorname{tr}\left(\mathrm{BCRB}_{\mathbf{h}_{1}}\right) \\
\text { s.t. } & \mathbf{X}^{H} \mathbf{X}=\rho_{s} \mathbf{I}_{L}, \\
\min _{\mathbf{X}, \mathbf{T}_{r}} & \operatorname{tr}\left(\mathrm{BCRB}_{\mathbf{h}_{2}}\right) \\
\text { s.t. } & \mathbf{X}^{H} \mathbf{X}=\rho_{s} \mathbf{I}_{L} \\
& \mathbf{T}_{r}^{H} \mathbf{T}_{r}=\rho_{t} \mathbf{I}_{L} .
\end{array}
$$

Lemma 2. The training sequences satisfying

$$
\begin{gathered}
\mathbf{X}^{*} \mathbf{X}^{T}=\frac{L}{N} \rho_{s} \mathbf{I}_{N}, \\
\left(\mathbf{T}_{r}^{*} \otimes \mathbf{I}_{M}\right)\left(\mathbf{T}_{r}^{T} \otimes \mathbf{I}_{M}\right)=\frac{L}{R} \rho_{t} \mathbf{I}_{M R},
\end{gathered}
$$

are optimal solutions to $(P 1)$ and $(P 2)$.

Proof. See Appendix B.

The BCRBs become diagonal matrices with the optimal training in $(C 1)$ and $(C 2)$, and the bounds for MSEs of individual channel taps can be obtained as

$$
\begin{aligned}
\operatorname{MSE}_{h_{1 i}} & =E\left\{\left|\Delta h_{1 i}\right|^{2}\right\} \geq \frac{1}{\left(c_{1} \rho_{s}+\left(1 / \sigma_{h_{1}, i}^{2}\right)\right)}, \\
\operatorname{MSE}_{h_{2 i}} & =E\left\{\left|\Delta h_{2 i}\right|^{2}\right\} \\
& \geq \frac{1}{\left(c_{2} L \rho_{s}+c_{3}(L / R) \rho_{t}+c_{4}+\left(1 / \sigma_{h_{2}, i}^{2}\right)\right)} .
\end{aligned}
$$

\section{Suboptimal Channel Estimation Algorithm}

Using the optimal training vectors, we refer to the suboptimal estimators to verify the Bayesian Cramér-Rao Lower Bound 
(CRLB) results in the MIMO AF OWRN, since the channel statistics are assumed known. A linear minimum mean square error (LMMSE) estimator is designed to estimate the two sets of MIMO channels. The corresponding covariances $R_{\mathrm{H}}$ and $R_{\mathrm{N}}$ are given as below, and the calculations are listed in Appendix C,

$$
\begin{gathered}
R_{\mathbf{H}_{2}}=E\left\{\mathbf{H}_{2}^{H} \mathbf{H}_{2}\right\}=M \mathbf{I}_{R}, \\
R_{\mathbf{H}}=E\left\{\mathbf{H}^{H} \mathbf{H}\right\}=\frac{\alpha^{2} \rho_{r}}{R\left(1+\rho_{s}\right)} E\left\{\mathbf{H}_{1}^{H} R_{\mathbf{H}_{2}} \mathbf{H}_{1}\right\}=\frac{\alpha^{2} \rho_{r} M}{\left(1+\rho_{s}\right)} \mathbf{I}_{N} .
\end{gathered}
$$

Recalling $\mathbf{N}=\alpha \mathbf{H}_{2} \mathbf{G} \mathbf{W}_{R}+\mathbf{W}_{D}$, and $\mathbf{W}_{R}$ and $\mathbf{W}_{D}$ are mutually independent, we have

$$
R_{\mathbf{N}}=E\left\{\mathbf{N}^{H} \mathbf{N}\right\}=\frac{M\left(1+\rho_{s}+\alpha^{2} \rho_{r}\right)}{\left(1+\rho_{s}\right)} \mathbf{I}_{L} .
$$

The LMMSE estimate of $\mathbf{H}$ is expressed as

$$
\hat{\mathbf{H}}=\mathbf{Y}\left(\mathbf{X}^{H} R_{\mathbf{H}} \mathbf{X}+\mathbf{T}_{r}^{H} R_{\mathbf{H}_{2}} \mathbf{T}_{r}+R_{\mathbf{N}}\right)^{-1} \mathbf{X}^{H} R_{\mathbf{H}},
$$

and the corresponding estimate error can be computed as

$$
\mathrm{MSE}=\operatorname{tr}\left\{\left(R_{\mathbf{H}}^{-1}+\mathbf{X}\left(\mathbf{T}_{r}^{H} R_{\mathbf{H}_{2}} \mathbf{T}_{r}+R_{\mathbf{N}}\right)^{-1} \mathbf{X}^{H}\right)^{-1}\right\} .
$$

Similarly, the LMMSE estimate of $\mathbf{H}_{2}$ is expressed as

$$
\hat{\mathbf{H}}_{2}=\mathbf{Y}\left(\mathbf{X}^{H} R_{\mathbf{H}} \mathbf{X}+\mathbf{T}_{r}^{H} R_{\mathbf{H}_{2}} \mathbf{T}_{r}+R_{\mathbf{N}}\right)^{-1} \mathbf{T}_{r}^{H} R_{\mathbf{H}_{2}},
$$

and the corresponding estimate error can be computed as

$$
\mathrm{MSE}=\operatorname{tr}\left\{\left(R_{\mathbf{H}_{2}}^{-1}+\mathbf{T}_{r}\left(\mathbf{X}^{H} R_{\mathbf{H}} \mathbf{X}+R_{\mathbf{N}}\right)^{-1} \mathbf{T}_{r}^{H}\right)^{-1}\right\} .
$$

With $\hat{\mathbf{H}}$ and $\hat{\mathbf{H}}_{2}$, the estimate of $\mathbf{H}_{1}$ can be computed as

$$
\hat{\mathbf{H}}_{1}=\left(\alpha \mathbf{H}_{2} \mathbf{G}\right)^{\dagger} \hat{\mathbf{H}} \text {. }
$$

Other estimation algorithms can always be applied to achieve better MSE performance with additional computational complexity accompanied, and here we give an example of modified LMMSE algorithm. Since $\hat{\mathbf{H}}_{2}$ can be directly obtained from $\mathbf{Y}$, we can further exploit $\hat{\mathbf{H}}_{2}$ for the estimation of $\hat{\mathbf{H}}_{1}$, that is, remove the impact of $\hat{\mathbf{H}}_{2}$ over $\hat{\mathbf{H}}_{1}$ from $\mathbf{Y}$ according to the interference cancellation principle, and take into account the fact that the overall noise component at the destination contains certain information about $\hat{\mathbf{H}}_{2}$.

The modified estimation process is as follows, after obtaining the LMMSE estimate $\hat{\mathbf{H}}_{2}$, the received signal can be rewritten as

$$
\begin{aligned}
\mathbf{y}-\left(\mathbf{T}_{r}^{T} \otimes \mathbf{I}_{M}\right) \hat{\mathbf{h}}_{2}= & \mathscr{H}_{2} \mathbf{h}_{1}+\alpha\left(\mathbf{I}_{L} \otimes \hat{\mathbf{H}}_{2} \mathbf{G}\right) \operatorname{vec}\left(\mathbf{W}_{R}\right) \\
& +\operatorname{vec}\left(\mathbf{W}_{D}\right)
\end{aligned}
$$

where $\mathscr{H}_{2}=\alpha\left[\mathbf{X}^{T} \otimes\left(\hat{\mathbf{H}}_{2} \mathbf{G}\right)\right]$, then the LMMSE estimate of $\mathbf{h}_{1}$ can be computed as

$$
\begin{aligned}
\hat{\mathbf{h}}_{1}= & R_{\mathbf{h}_{1}} \mathscr{H}_{2}^{H} \\
\times & \quad\left[\mathscr{H}_{2} R_{\mathbf{h}_{1}} \mathscr{H}_{2}^{H}\right. \\
& \left.\quad+\sigma_{n}^{2}\left(\alpha^{2}\left(\mathbf{I}_{L} \otimes \hat{\mathbf{H}}_{2} \mathbf{G}\right)\left(\mathbf{I}_{L} \otimes \hat{\mathbf{H}}_{2} \mathbf{G}\right)^{H}+\mathbf{I}_{L M}\right)\right]^{-1} \\
& \times\left(\mathbf{y}-\left(\mathbf{T}_{r}^{T} \otimes \mathbf{I}_{M}\right) \hat{\mathbf{h}}_{2}\right),
\end{aligned}
$$

where $R_{\mathbf{h}_{1}}=\mathbf{I}_{R N}$.

\section{Simulations}

In this section, we provide numerical results to verify our studies. For the sake of simplicity and without loss of generality, we set $N=M=R=2, T=64, \rho_{s}=$ $\rho_{r}$, and $\sigma_{n}^{2}=1$. The optimal training sequences designed from the Bayesian CRLBs are used, and the total training power is the same in all the scenarios. The NMSE is defined as the channel estimation MSE divided by the product of the number of transmit antennas and receive antennas, for example, $\mathrm{NMSE}_{\mathrm{H}_{1}}=\mathrm{MSE}_{\mathrm{H}_{1}} / R N, \mathrm{NMSE}_{\mathrm{H}_{2}}=\mathrm{MSE}_{\mathrm{H}_{2}} / M R$.

The theoretical Bayesian CRLBs are then calculated for $\mathbf{H}_{1}$ and $\mathbf{H}_{2}$ as the function of $\alpha$ and SNR, as in Figures 2 and 3 , respectively. It is observed that the Bayesian CRLB of $\mathbf{H}_{2}$ is always smaller than that of $\mathbf{H}_{1}$ for any given $\alpha$, which is expected because the superimposed training is devoted to the estimation of $\mathbf{H}_{2}$ while the training sequences from the source contribute the same power to the estimation of both $\mathbf{H}_{1}$ and $\mathbf{H}_{2}$. Moreover, as $\alpha$ increases, the power allocated for the original training from the source becomes greater, which means that the training power for the estimation of $\mathbf{H}_{\mathbf{1}}$ becomes larger, thus the NMSE performance is improved and the Bayesian CRLB of $\mathbf{H}_{\mathbf{l}}$ gets lower accordingly. Meanwhile, the training for the estimation of $\mathbf{H}_{2}$ comes from both the original training from the source and the superimposed training from the relay, so the training power is always balanced automatically, and thus the Bayesian CRLB of $\mathbf{H}_{2}$ maintains at a stable level regardless of the values of $\alpha$.

We then examine the NMSE performance of the suboptimal estimator, as shown together with the theoretical Bayesian CRLBs in Figures 4, 5, and 6 for different values of $\alpha$. Since $\mathbf{H}_{1}$ is obtained by removing the effect of $\mathbf{H}_{2}$, where the error in the estimated $\mathbf{H}_{2}$ should be taken into account, the NMSE performance of channel estimation for $\mathbf{H}_{2}$ is always better than that for $\mathbf{H}_{1}$, which is consistent with the comparison between the two Bayesian CRLBs. Moreover, as $\alpha$ increases, the power allocated for the superimposed training from the relay becomes smaller; thus, the direct estimate of $\mathbf{H}_{2}$ is deteriorated accordingly, which can be seen from the increasing gap between the channel estimation NMSEs and the Bayesian CRLBs of $\mathbf{H}_{2}$. As for the estimation of $\mathbf{H}_{1}$, although the power allocated for the original training from the source becomes greater as $\alpha$ increases, the error effect from the estimation of $\mathbf{H}_{2}$ also becomes greater, and 


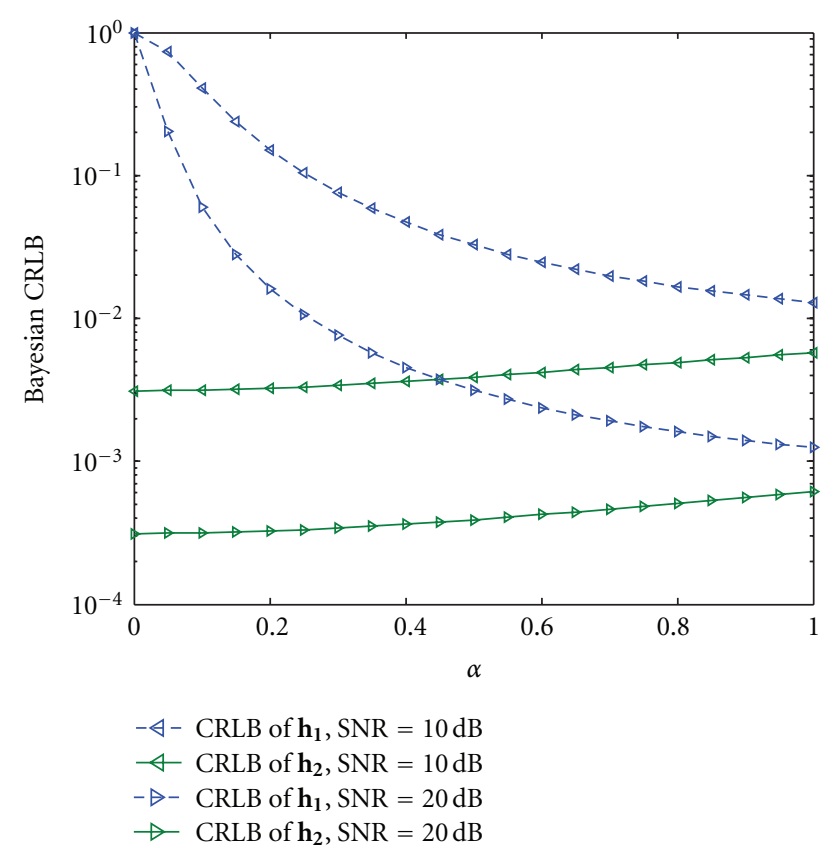

FIgURE 2: Bayesian CRLBs versus $\alpha$.

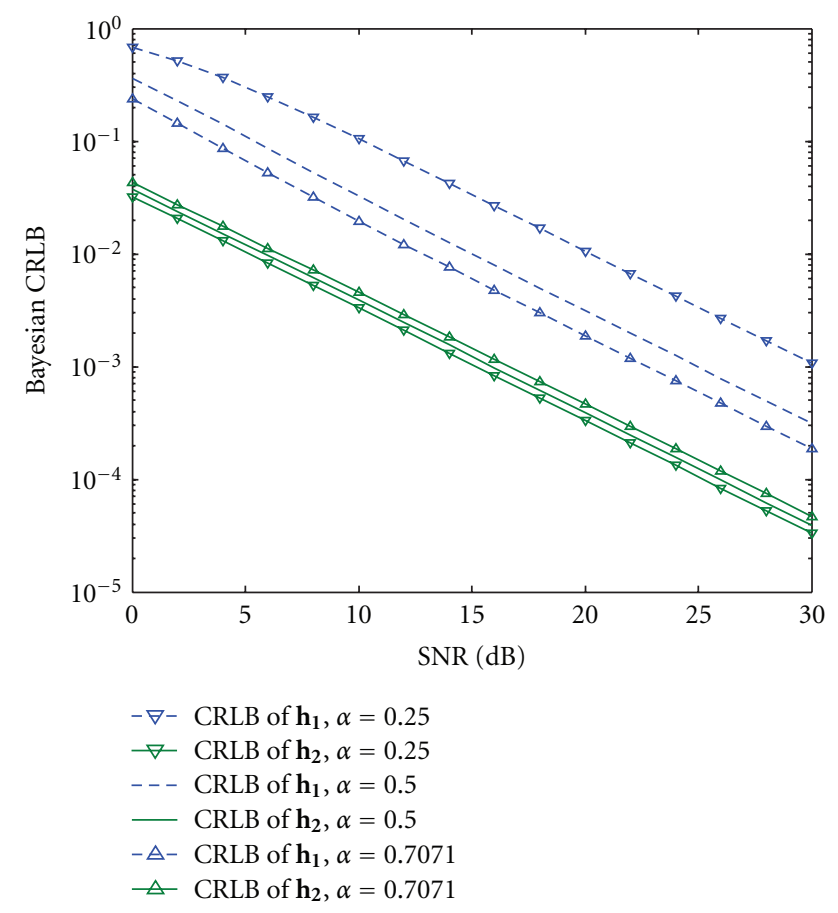

FIgure 3: Bayesian CRLBs versus SNR.

thus the gap between the channel estimation NMSEs and the Bayesian CRLBs of $\mathbf{H}_{\mathbf{1}}$ remains basically unchanged for different values of $\alpha$.

We also provide the simulation results considering different numbers of antennas at the relay and destination, for example, same $N$, different $R$ and $M$ in Figure 7 with $\alpha$ fixed as 0.5 . It is seen that when the same transmit power

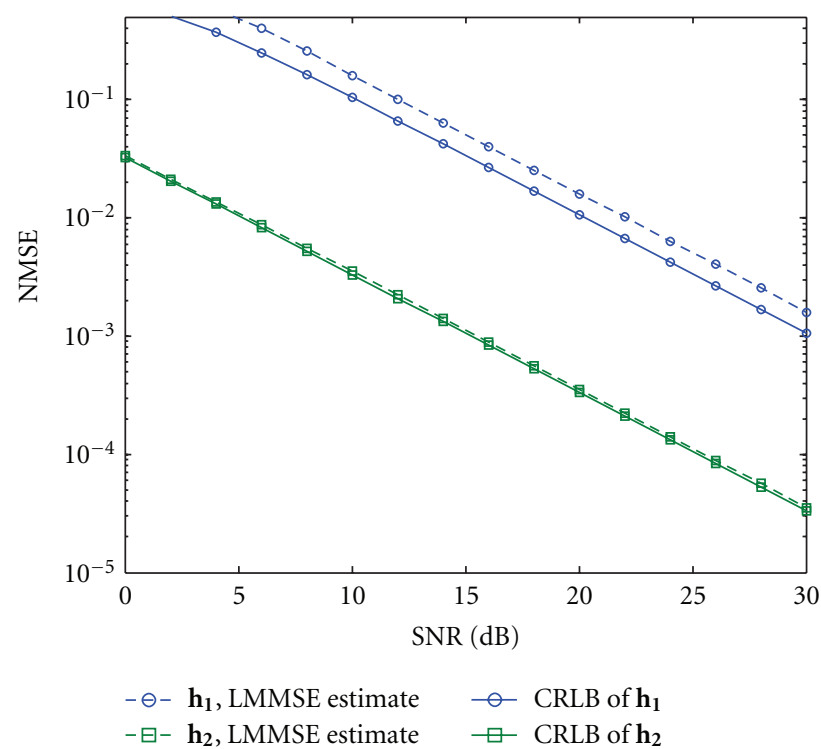

FIgure 4: Channel estimation NMSEs versus SNR for $\alpha=0.25$.

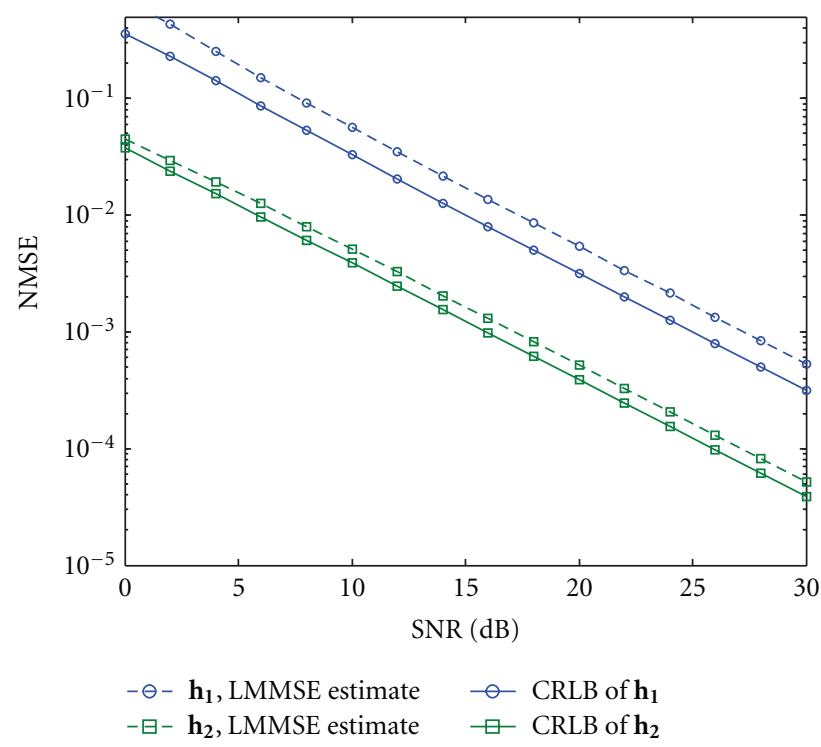

FIGURE 5: Channel estimation NMSEs versus SNR for $\alpha=0.5$.

is applied for the source and relay, the antenna number affects the channel estimation in MIMO AF OWRN a lot. The channel estimation NMSE decreases when $M$, the number of receive antennas at the destination, increases, especially the NMSE of $\mathbf{H}_{1}$, which is a consistent phenomenon as in the traditional MIMO channel estimation. On the other hand, the channel estimation NMSE increases when $R$, the number of transmit antennas at the relay, increases. Note that, in the traditional multiple-input single-output systems, the channel estimation MSE is only related with the transmit power but is not related with the number of antennas. However, the reason for the degrading performance in MIMO AF relay networks is because the relay forwards additional noise to the destination too, while the total training power remains the same. 


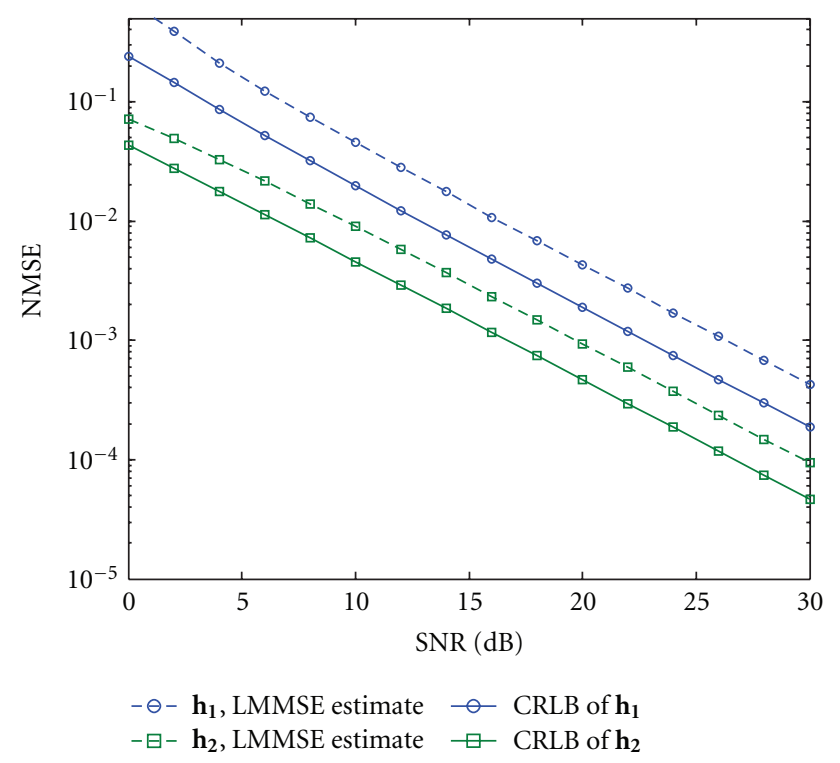

FIgURE 6: Channel estimation NMSEs versus SNR for $\alpha=0.7071$.

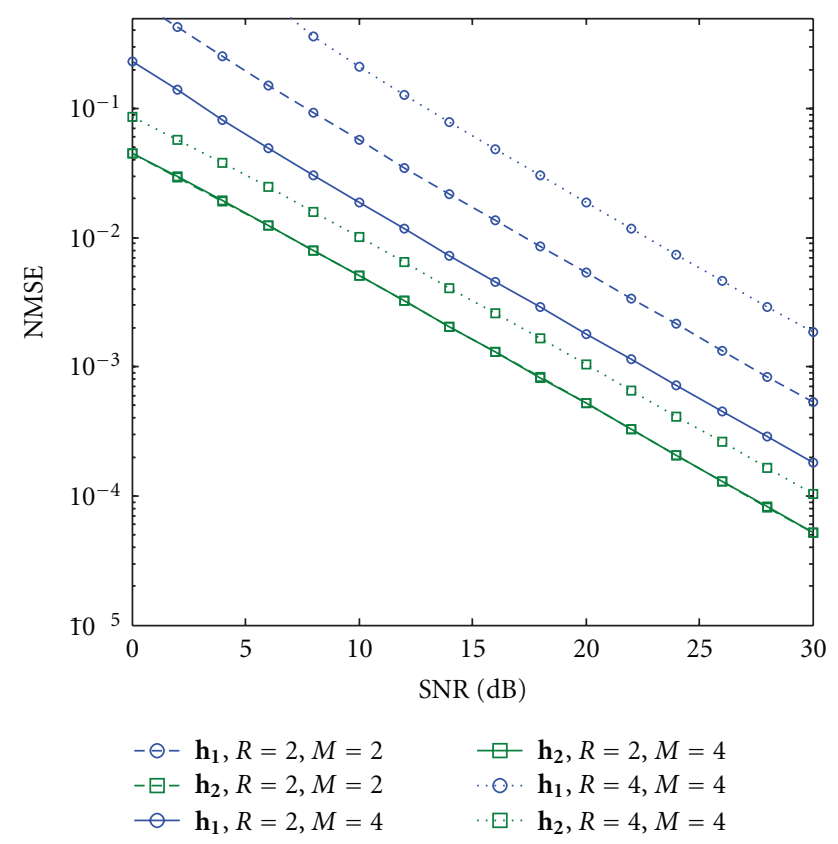

FIgURE 7: Channel estimation NMSEs versus $R$ and $M$ for $\alpha=0.5$.

The NMSE performance of the modified LMMSE algorithm is depicted in Figure 8 with $\alpha$ fixed as 0.5. As we can see, the estimated $\mathbf{H}_{1}$ could directly benefit from the interference cancellation and the information of $\mathbf{H}_{2}$ contained in the noise covariance, especially in low SNR region. The gap between the estimation NMSEs and their corresponding CRLBs is caused by the utilization of lowcomplexity LMMSE estimator instead of the optimal MMSE or MAP estimator.

The simulation results verify our proposal that with a fraction of relay power devoted to the superimposed training

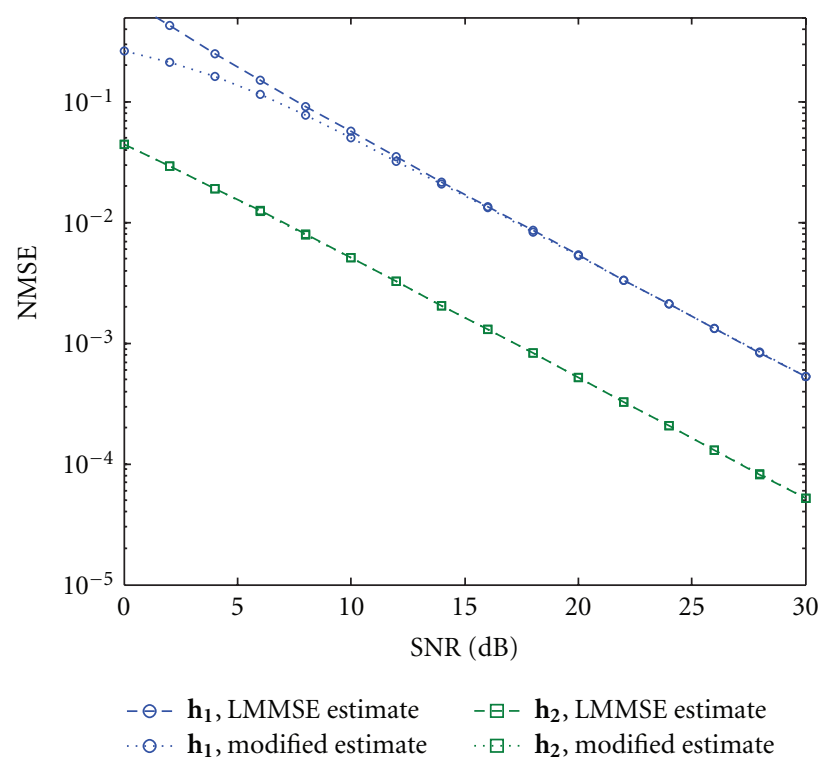

FIgure 8: Modified LMMSE channel estimation NMSEs versus SNR for $\alpha=0.5$.

in the MIMO AF OWRN, the individual channel information of $\mathbf{H}_{1}$ and $\mathbf{H}_{2}$ can be directly obtained at the destination, and the channel estimation of $\mathbf{H}_{1}$ and $\mathbf{H}_{2}$ can be simplified while the corresponding NMSE performance maintains the same level as the conventional channel estimation in the case of the same power. Furthermore, we can expect that, the estimates in our proposal can be further improved, especially the estimate of $\mathbf{H}_{1}$, if the relay power in the MIMO AF OWRN can be increased, since more power can be allocated to the training sequences at the relay.

\section{Conclusion}

In this paper, we have investigated the superimposed training strategy in MIMO AF TWRN, which superimposes a new set of training vectors at the relay and provides the individual channel information at the destination to accomplish and simplify the channel estimation. The closed-form Bayesian CRLB has been derived and then used to guide the optimal training design in MIMO AF OWRN. The simulation results have been provided to verify the Bayesian CRLB results by the practical NMSE performance of a suboptimal channel estimator. With the individual channel information, the joint optimization of the throughout, as well as the tradeoff between rate and detection, can be achieved at the destination.

\section{Appendices}

\section{A. Proof of Lemma 1}

The FIM can be modified as 


$$
\begin{aligned}
\mathscr{g}= & E\left\{\frac{\partial \ln p(\mathbf{Y}, \theta)}{\partial \theta^{*}}\left(\frac{\partial \ln p(\mathbf{Y}, \theta)}{\partial \theta^{*}}\right)^{H}\right\} \\
= & E_{\theta}\left\{E_{\mathbf{Y} \mid \theta}\left\{\frac{\partial \ln p(\mathbf{Y}, \theta)}{\partial \theta^{*}}\left(\frac{\partial \ln p\left(\mathbf{y}_{1}, \theta\right)}{\partial \theta^{*}}\right)^{H} \mid \theta\right\}\right\} \\
= & E_{\theta}\left\{E_{\mathbf{Y} \mid \theta}\left\{\frac{\partial \ln p(\mathbf{Y} \mid \theta)}{\partial \theta^{*}}\left(\frac{\partial \ln p\left(\mathbf{y}_{1} \mid \theta\right)}{\partial \theta^{*}}\right)^{H}\right\}\right. \\
+ & \left.\frac{\partial \ln p(\theta)}{\partial \theta^{*}}\left(\frac{\partial \ln p(\theta)}{\partial \theta^{*}}\right)^{H}\right\}
\end{aligned}
$$

where the first and the second expectations are over $p(\theta)$ and $p(\mathbf{Y} \mid \theta)$, respectively.

Since $\mathbf{h}_{1}$ and $\mathbf{h}_{2}$ are mutually independent, we have $p(\theta)=p\left(\mathbf{h}_{1}\right) p\left(\mathbf{h}_{2}\right)$, and the second term in (A.1) becomes

$$
E_{\theta}\left\{\frac{\partial \ln p(\theta)}{\partial \theta^{*}}\left(\frac{\partial \ln p(\theta)}{\partial \theta^{*}}\right)^{H}\right\}=\left[\begin{array}{cc}
\mathbf{R}_{\mathbf{h}_{1}}^{-1} & \mathbf{0}_{L_{h} \times L_{g}} \\
\mathbf{0}_{L_{g} \times L_{h}} & \mathbf{R}_{\mathbf{h}_{2}}^{-1}
\end{array}\right] .
$$

Denote $\mathbf{y}=\operatorname{vec}(\mathbf{Y})=\left[\mathbf{Y}(1)^{T}, \ldots, \mathbf{Y}(L)^{T}\right]^{T}$, and $\mathbf{n}=$ $\operatorname{vec}(\mathbf{N})=\left[\mathbf{w}(1)^{T}, \ldots, \mathbf{w}(L)^{T}\right]^{T}$. Conditioned on $\theta$, the mean value of $\mathbf{y}$ can be defined as

$$
\begin{aligned}
\mu(\theta) & =\operatorname{vec}\left(\alpha \mathbf{H}_{2} \mathbf{G H}_{1} \mathbf{X}+\mathbf{H}_{2} \mathbf{T}_{r}\right) \\
& =\operatorname{vec}\left(\alpha \mathbf{H}_{2} \mathbf{G H}_{1} \mathbf{X}\right)+\operatorname{vec}\left(\mathbf{H}_{2} \mathbf{T}_{r}\right) \\
& =\alpha\left[\mathbf{X}^{T} \otimes\left(\mathbf{H}_{2} \mathbf{G}\right)\right] \mathbf{h}_{1}+\left(\mathbf{T}_{r}^{T} \otimes \mathbf{I}_{M}\right) \mathbf{h}_{2},
\end{aligned}
$$

or equivalently

$$
\begin{aligned}
\mu(\theta) & =\alpha\left(\left(\mathbf{G H}_{1} \mathbf{X}\right)^{T} \otimes \mathbf{I}_{M}\right) \mathbf{h}_{2}+\left(\mathbf{T}_{r}^{T} \otimes \mathbf{I}_{M}\right) \mathbf{h}_{2} \\
& =\left[\alpha\left(\mathbf{G H}_{1} \mathbf{X}\right)^{T} \otimes \mathbf{I}_{M}+\mathbf{T}_{r}^{T} \otimes \mathbf{I}_{M}\right] \mathbf{h}_{2} .
\end{aligned}
$$

Then the received signal at the destination after vectorizing can be rewritten as

$$
\mathbf{y}=\alpha\left[\mathbf{X}^{T} \otimes\left(\mathbf{H}_{2} \mathbf{G}\right)\right] \mathbf{h}_{1}+\left(\mathbf{T}_{r}^{T} \otimes \mathbf{I}_{M}\right) \mathbf{h}_{2}+\mathbf{n},
$$

or equivalently

$$
\mathbf{y}=\left[\alpha\left(\mathbf{G H}_{1} \mathbf{X}\right)^{T} \otimes \mathbf{I}_{M}+\mathbf{T}_{r}^{T} \otimes \mathbf{I}_{M}\right] \mathbf{h}_{2}+\mathbf{n} .
$$

For computational simplicity, we further define

$$
\mathcal{F}=E_{\mathbf{y} \mid \theta}\left\{\frac{\partial \ln p(\mathbf{y} \mid \theta)}{\partial \theta^{*}}\left(\frac{\partial \ln p(\mathbf{y} \mid \theta)}{\partial \theta^{*}}\right)^{H}\right\}\left[\begin{array}{cc}
\mathcal{F}_{11} & \mathcal{F}_{12} \\
\mathcal{F}_{12}^{H} & \mathcal{F}_{22}
\end{array}\right],
$$

which is the FIM for the deterministic $\theta$.

The log-likelihood function of the received signal at the destination can be written as

$$
p(\mathbf{y} \mid \theta)=\frac{1}{\pi^{N} \mid \mathbf{R}_{\mathbf{n}|\theta|}} \exp \left\{-(\mathbf{y}-\mu)^{H} \mathbf{R}_{\mathbf{n} \mid \theta}^{-1}(\mathbf{y}-\mu)\right\} .
$$

The $(i, j)$ th element of the deterministic FIM is computed as

$$
[\mathcal{F}]_{i j}=\left(\frac{\partial \mu}{\partial \theta_{i}^{*}}\right)^{H} \mathbf{R}_{\mathbf{n} \mid \theta}^{-1}\left(\frac{\partial \mu}{\partial \theta_{j}}\right)+\operatorname{tr}\left(\mathbf{R}_{\mathbf{n} \mid \theta}^{-1} \frac{\partial \mathbf{R}_{\mathbf{n} \mid \theta}}{\partial \theta_{i}^{*}} \mathbf{R}_{\mathbf{n} \mid \theta}^{-1} \frac{\partial \mathbf{R}_{\mathbf{n} \mid \theta}}{\partial \theta_{j}}\right) .
$$

Conditioned on a specific channel realization, the noise term in $\mathbf{Y}$ is AWGN with variance $\mathbf{R}_{\mathbf{n} \mid \theta}$,

$$
\begin{aligned}
\mathbf{R}_{\mathbf{n} \mid \theta} & =\operatorname{vec}(\mathbf{N})(\operatorname{vec}(\mathbf{N}))^{H} \\
& =\mathbf{I}_{L} \otimes\left[\sigma_{n}^{2}\left(\alpha^{2} \mathbf{H}_{2} \mathbf{G G}^{H} \mathbf{H}_{2}^{H}+\mathbf{I}_{M}\right)\right] \\
& \triangleq \mathbf{I}_{L} \otimes \mathbf{C},
\end{aligned}
$$

where

$$
\mathbf{C}=\sigma_{n}^{2}\left(\alpha^{2} \mathbf{H}_{2} \mathbf{G G}^{H} \mathbf{H}_{2}^{H}+\mathbf{I}_{M}\right) .
$$

It can be obtained that

$$
\begin{gathered}
\frac{\partial \mu}{\partial \mathbf{h}_{1}}=\alpha \mathbf{X}^{T} \otimes\left(\mathbf{H}_{2} \mathbf{G}\right), \\
\frac{\partial \mu}{\partial \mathbf{h}_{2}}=\alpha\left(\mathbf{G} \mathbf{H}_{1} \mathbf{X}\right)^{T} \otimes \mathbf{I}_{M}+\mathbf{T}_{r}^{T} \otimes \mathbf{I}_{M}, \\
\frac{\partial \mathbf{R}_{\mathbf{n} \mid \theta}}{\partial h_{2 i}^{*}}=\mathbf{I}_{L} \otimes\left(\sigma_{n}^{2} \alpha^{2} \mathbf{H}_{2} \mathbf{G G}^{H} \frac{\partial \mathbf{H}_{2}^{H}}{\partial h_{2 i}^{*}}\right) \\
=\mathbf{I}_{L} \otimes\left(\sigma_{n}^{2} \alpha^{2} \mathbf{H}_{2} \mathbf{G G}^{H}\left(\operatorname{unvec}_{M, R}\left(\mathbf{e}_{i}\right)\right)^{H}\right) \\
=\mathbf{I}_{L} \otimes\left(\sigma_{n}^{2} \alpha^{2} \mathbf{H}_{2} \mathbf{G G}^{H} \mathbf{M}_{i}^{H}\right) \\
=\mathbf{I}_{L} \otimes\left(\sigma_{n}^{2} \alpha^{2} \mathbf{M}_{i} \mathbf{G G}^{H} \mathbf{H}_{2}^{H}\right),
\end{gathered}
$$

where $\mathbf{M}_{i}=\left(\partial \mathbf{H}_{2} / \partial h_{2 i}\right)=\operatorname{unvec}_{M, R}\left(\mathbf{e}_{i}\right)$. Define a new matrix $\mathbf{Q}$ whose $(i, j)$ th entry is the second term on the RHS of $[\mathcal{F}]_{i j}$,

$$
\begin{aligned}
{[\mathbf{Q}]_{i j}=} & \alpha^{4} \sigma_{n}^{4} L \operatorname{tr}\left\{\mathbf{C}^{-1}\left(\mathbf{H}_{2} \mathbf{G} \mathbf{G}^{H} \mathbf{M}_{i}^{H}\right) \mathbf{C}^{-1}\left(\mathbf{M}_{j} \mathbf{G G}^{H} \mathbf{H}_{2}^{H}\right)\right\} \\
= & \frac{\alpha^{4} \sigma_{n}^{4} L \rho_{r}^{2}}{R^{2}\left(1+\rho_{s}\right)^{2}} \operatorname{tr}\left\{\mathbf{C}^{-1}\left(\mathbf{H}_{2} \mathbf{M}_{i}^{H}\right) \mathbf{C}^{-1}\left(\mathbf{M}_{j} \mathbf{H}_{2}^{H}\right)\right\} \\
= & \frac{\alpha^{4} \sigma_{n}^{4} L \rho_{r}^{2}}{R^{2}\left(1+\rho_{s}\right)^{2}}\left(\operatorname{vec}\left(\left(\mathbf{M}_{j} \mathbf{H}_{2}^{H}\right)^{T}\right)\right)^{T}\left(\mathbf{C}^{-T} \otimes \mathbf{C}^{-1}\right) \\
& \times \operatorname{vec}\left(\mathbf{H}_{2} \mathbf{M}_{i}^{H}\right) .
\end{aligned}
$$

Let $\mathcal{M}=\left[\operatorname{vec}\left(\mathbf{H}_{2} \mathbf{M}_{1}^{H}\right), \operatorname{vec}\left(\mathbf{H}_{2} \mathbf{M}_{2}^{H}\right), \ldots, \operatorname{vec}\left(\mathbf{H}_{2} \mathbf{M}_{P}^{H}\right)\right]$, where $P$ is the length of the vector $\mathbf{h}_{2}^{T}$, and then $\mathbf{Q}$ could be expressed in a compact form

$$
\mathbf{Q}=\frac{\alpha^{4} \sigma_{n}^{4} L \rho_{r}^{2}}{R^{2}\left(1+\rho_{s}\right)^{2}} \mathcal{M}^{H}\left(\mathbf{C}^{-T} \otimes \mathbf{C}^{-1}\right) \mathcal{M} .
$$


Note that the columns of $\mathcal{M}$ are orthogonal to each other.

$\mathcal{F}_{11}$ can be obtained as

$$
\begin{aligned}
\mathcal{F}_{11} & =\alpha^{2}\left[\mathbf{X}^{*} \otimes\left(\mathbf{G}^{H} \mathbf{H}_{2}^{H}\right)\right] \mathbf{R}_{\mathbf{n} \mid \theta}^{-1}\left[\mathbf{X}^{T} \otimes\left(\mathbf{H}_{2} \mathbf{G}\right)\right] \\
& =\frac{\alpha^{2} \rho_{r}}{R\left(1+\rho_{s}\right)}\left(\mathbf{X}^{*} \otimes \mathbf{H}_{2}^{H}\right) \mathbf{R}_{\mathbf{n} \mid \theta}^{-1}\left(\mathbf{X}^{T} \otimes \mathbf{H}_{2}\right) .
\end{aligned}
$$

Note that $\left(\partial \mathbf{R}_{\mathbf{n} \mid \theta} / \partial h_{1 i}^{*}\right)=0$. Following a similar approach, we obtain

$$
\begin{gathered}
\mathcal{F}_{12}=\alpha\left[\mathbf{X}^{*} \otimes\left(\mathbf{G}^{H} \mathbf{H}_{2}^{H}\right)\right] \mathbf{R}_{\mathbf{n} \mid \theta}^{-1}\left[\alpha\left(\mathbf{G H}_{1} \mathbf{X}\right)^{T} \otimes \mathbf{I}_{M}+\mathbf{T}_{r}^{T} \otimes \mathbf{I}_{M}\right], \\
\mathcal{F}_{21}=\alpha\left[\alpha\left(\mathbf{G} \mathbf{H}_{1} \mathbf{X}\right)^{*} \otimes \mathbf{I}_{M}+\mathbf{T}_{r}^{*} \otimes \mathbf{I}_{M}\right] \mathbf{R}_{\mathbf{n} \mid \theta}^{-1}\left[\mathbf{X}^{T} \otimes\left(\mathbf{H}_{2} \mathbf{G}\right)\right], \\
\mathcal{F}_{22}=\left[\alpha\left(\mathbf{G H}_{1} \mathbf{X}\right)^{*} \otimes \mathbf{I}_{M}+\mathbf{T}_{r}^{*} \otimes \mathbf{I}_{M}\right] \mathbf{R}_{\mathbf{n} \mid \theta}^{-1} \\
\times\left[\alpha\left(\mathbf{G H}_{1} \mathbf{X}\right)^{T} \otimes \mathbf{I}_{M}+\mathbf{T}_{r}^{T} \otimes \mathbf{I}_{M}\right]+Q .
\end{gathered}
$$

The Bayesian FIM $g$ can be found from averaging $\mathcal{F}$ over $\theta$.

It is assumed that there is no spatial correlation at the relay. According to the widely used Kronecker correlation model $[24,25]$, let $\mathbf{R}_{s}$ and $\mathbf{R}_{d}$ be the spatial correlation matrices (positive semidefinite and Hermitian) at the source and destination, then $\mathbf{H}_{1}$ and $\mathbf{H}_{2}$ can be expressed as $\mathbf{H}_{w 1} \mathbf{R}_{s}^{1 / 2}$ and $\mathbf{R}_{d}^{1 / 2} \mathbf{H}_{w 2}$, respectively, where $\mathbf{H}_{w 1}$ and $\mathbf{H}_{w 2}$ are $R \times N$ and $M \times R$ independent and identically distributed (i.i.d.) ZMCSCG matrices. Note that $\operatorname{tr}\left(\mathbf{R}_{s}\right)=N$ and $\operatorname{tr}\left(\mathbf{R}_{d}\right)=M$ for normalization and that $\mathbf{H}_{w 1}$ and $\mathbf{H}_{w 2}$ are independent mutually which means that the backward link $\mathbf{H}_{1}$ is independent from the forward link $\mathbf{H}_{2}$. Moreover, every Hermitian matrix is a normal matrix, and the finite-dimensional spectral theorem applies. It says that any Hermitian matrix can be diagonalized by a unitary matrix, and that the resulting diagonal matrix has only real entries. This implies that all eigenvalues of a Hermitian matrix $\mathbf{A}$ are real, and that $\mathbf{A}$ has $n$ linearly independent eigenvectors. The EVD of $\mathbf{H}_{2} \mathbf{H}_{2}^{H}$ is denoted as $\mathbf{U}_{2} \mathbf{D}_{2} \mathbf{U}_{2}^{H}$, where $\mathbf{D}_{2}=\operatorname{diag}\left\{\lambda_{1}, \lambda_{2}, \ldots, \lambda_{M}\right\}$, and $\mathbf{U}_{2}$ is a unitary matrix.

From the above properties, it can then be calculated that

$$
\begin{aligned}
E_{\theta}\{\mathbf{Q}\} & =\frac{\alpha^{4} \sigma_{n}^{4} L \rho_{r}^{2}}{R^{2}\left(1+\rho_{s}\right)^{2}} \mathrm{E}_{\theta}\left\{\mathcal{M}^{H}\left(\mathbf{C}^{-T} \otimes \mathbf{C}^{-1}\right) \mathcal{M}\right\} \\
& =\frac{\alpha^{4} L M \rho_{r}^{2}\left[\left(1+\rho_{s}\right)+\sigma_{n}^{2}\left(1+\rho_{s}+\alpha^{2} \rho_{r}\right)\right]}{R^{2}\left(1+\rho_{s}\right)\left(1+\rho_{s}+\alpha^{2} \rho_{r}\right)^{2}} \mathbf{I}_{M R}, \\
E_{\theta}\left\{\mathcal{F}_{11}\right\} & =\mathrm{E}_{\theta}\left\{\frac{\alpha^{2} \rho_{r}}{R\left(1+\rho_{s}\right)}\left(\mathbf{X}^{*} \otimes \mathbf{H}_{2}^{H}\right) \mathbf{R}_{\mathbf{n} \mid \theta}^{-1}\left(\mathbf{X}^{T} \otimes \mathbf{H}_{2}\right)\right\} \\
& =\frac{\alpha^{2} \rho_{r}}{R\left(1+\rho_{s}\right)} \mathrm{E}_{\theta}\left\{\left(\mathbf{X}^{*} \mathbf{X}^{T}\right) \otimes\left(\mathbf{H}_{2}^{H} \mathbf{C}^{-1} \mathbf{H}_{2}\right)\right\} \\
& =\frac{\alpha^{2} \rho_{r}}{R\left(1+\rho_{s}\right)}\left(\mathbf{X}^{*} \mathbf{X}^{T}\right) \otimes \mathrm{E}_{\theta}\left\{\mathbf{H}_{2}^{H} \mathbf{C}^{-1} \mathbf{H}_{2}\right\} \\
& =\frac{\alpha^{2} \rho_{r}}{\sigma_{n}^{2} R\left(1+\rho_{s}+\alpha^{2} \rho_{r}\right)}\left(\mathbf{X}^{*} \mathbf{X}^{T}\right) \otimes \mathbf{I}_{R},
\end{aligned}
$$

and $\mathrm{E}_{\theta}\left\{\mathcal{F}_{11}\right\}$ is an $N R \times N R$ matrix.

$$
\begin{aligned}
& E_{\theta}\left\{\mathcal{F}_{21}\right\}=\mathbf{0}_{N R \times M R}, \\
& E_{\theta}\left\{\mathcal{F}_{21}\right\}=\mathbf{0}_{M R \times N R},
\end{aligned}
$$

$E_{\theta}\left\{\mathcal{F}_{22}\right\}$

$$
\begin{aligned}
= & E_{\theta}\left\{\alpha^{2}\left[\left(\mathbf{G H}_{1} \mathbf{X}\right)^{*} \otimes \mathbf{I}_{M}\right] \mathbf{R}_{\mathbf{n} \mid \theta}^{-1}\left[\left(\mathbf{G H}_{1} \mathbf{X}\right)^{T} \otimes \mathbf{I}_{M}\right]\right\} \\
& +\left(\mathbf{T}_{r}^{*} \otimes \mathbf{I}_{M}\right) \mathrm{E}_{\theta}\left\{\mathbf{R}_{\mathbf{n} \mid \theta}^{-1}\right\}\left(\mathbf{T}_{r}^{T} \otimes \mathbf{I}_{M}\right)+\mathrm{E}_{\theta}\{\mathbf{Q}\} \\
= & \frac{\alpha^{2} \rho_{r}}{R\left(1+\rho_{s}\right)} \mathrm{E}_{\theta}\left\{\left[\left(\mathbf{H}_{1} \mathbf{X}\right)^{*}\left(\mathbf{H}_{1} \mathbf{X}\right)^{T}\right] \otimes \mathbf{C}^{-1}\right\} \\
& +\frac{\left(1+\rho_{s}\right)}{\sigma_{n}^{2}\left(1+\rho_{s}+\alpha^{2} \rho_{r}\right)}\left(\mathbf{T}_{r}^{*} \otimes \mathbf{I}_{M}\right)\left(\mathbf{T}_{r}^{T} \otimes \mathbf{I}_{M}\right)+\mathrm{E}_{\theta}\{\mathbf{Q}\} \\
= & \frac{\alpha^{2} \rho_{r}}{\sigma_{n}^{2} R\left(1+\rho_{s}+\alpha^{2} \rho_{r}\right)} \mathrm{E}_{\theta}\left\{\mathbf{H}_{1}^{*} \mathbf{X}^{*} \mathbf{X}^{T} \mathbf{H}_{1}^{T}\right\} \otimes \mathbf{I}_{M}
\end{aligned}
$$$$
+\frac{\left(1+\rho_{s}\right)}{\sigma_{n}^{2}\left(1+\rho_{s}+\alpha^{2} \rho_{r}\right)}\left(\mathbf{T}_{r}^{*} \otimes \mathbf{I}_{M}\right)\left(\mathbf{T}_{r}^{T} \otimes \mathbf{I}_{M}\right)+\mathrm{E}_{\theta}\{\mathbf{Q}\}
$$$$
=\frac{\alpha^{2} \rho_{r}}{\sigma_{n}^{2} R\left(1+\rho_{s}+\alpha^{2} \rho_{r}\right)} \operatorname{tr}\left(\mathbf{X}^{*} \mathbf{X}^{T}\right) \mathbf{I}_{M R}
$$$$
+\frac{\left(1+\rho_{s}\right)}{\sigma_{n}^{2}\left(1+\rho_{s}+\alpha^{2} \rho_{r}\right)}\left(\mathbf{T}_{r}^{*} \otimes \mathbf{I}_{M}\right)\left(\mathbf{T}_{r}^{T} \otimes \mathbf{I}_{M}\right)+\mathrm{E}_{\theta}\{\mathbf{Q}\}
$$$$
=\frac{\alpha^{2} \rho_{r}}{\sigma_{n}^{2} R\left(1+\rho_{s}+\alpha^{2} \rho_{r}\right)} \operatorname{tr}\left(\mathbf{X}^{*} \mathbf{X}^{T}\right) \mathbf{I}_{M R}
$$

$$
\begin{aligned}
& +\frac{\left(1+\rho_{s}\right)}{\sigma_{n}^{2}\left(1+\rho_{s}+\alpha^{2} \rho_{r}\right)}\left(\mathbf{T}_{r}^{*} \otimes \mathbf{I}_{M}\right)\left(\mathbf{T}_{r}^{T} \otimes \mathbf{I}_{M}\right) \\
& +\frac{\alpha^{4} L M \rho_{r}^{2}\left[\left(1+\rho_{s}\right)+\sigma_{n}^{2}\left(1+\rho_{s}+\alpha^{2} \rho_{r}\right)\right]}{R^{2}\left(1+\rho_{s}\right)\left(1+\rho_{s}+\alpha^{2} \rho_{r}\right)^{2}} \mathbf{I}_{M R}
\end{aligned}
$$

and $E_{\theta}\left\{\widetilde{F}_{22}\right\}$ is an $M R \times M R$ matrix.

\section{B. Proof of Lemma 2}

We first derive the optimal solutions for $(P 1)$. First observe that $\mathbf{R}_{\mathbf{h}_{1}}^{-1}$ is a constant diagonal matrix, and the diagonal elements of $\mathbf{X}^{*} \mathbf{X}^{T}$ are constant $\rho_{s}$. We know that for any positive-definite matrix $\mathbf{X}$, there is

$$
\operatorname{tr}\left(\mathbf{X}^{-1}\right) \geq \sum_{i=0}^{N-1} \frac{1}{[\mathbf{X}]_{i i}},
$$

where the equality holds when $\mathbf{X}$ is diagonal. Applying this inequality, we obtain

$$
\operatorname{tr}\left(\mathrm{BCRB}_{\mathbf{h}_{1}}\right) \geq \sum_{i=0}^{N R-1} \frac{1}{\left(c_{1} \rho_{s}+\left(1 / \sigma_{h_{1}, i}^{2}\right)\right)},
$$

where the RHS is not related with $\mathbf{X}$ and can immediately serve as the lower bound of $\operatorname{tr}\left(\mathrm{BCRB}_{\mathbf{h}_{1}}\right)$. The lower bound is achieved when $\mathbf{X}^{*} \mathbf{X}^{T}=(L / N) \rho_{s} \mathbf{I}_{N}$. Obviously, the choice $\left|\tilde{x}_{0}\right|^{2}=\cdots=\left|\tilde{x}_{N L-1}\right|^{2}=\rho_{s} / N$ satisfies the requirement. 
Similarly we can obtain the optimal solution for $(P 2)$. As for $(P 2), \mathbf{R}_{\mathbf{h}_{2}}^{-1}$ is a constant diagonal matrix, and the diagonal elements of $\left(\mathbf{T}_{r}^{*} \otimes \mathbf{I}_{M}\right)\left(\mathbf{T}_{r}^{T} \otimes \mathbf{I}_{M}\right)$ are cosnstant $(L / R) \rho_{t}$. Applying the inequality, we obtain

$$
\operatorname{tr}\left(\mathrm{BCRB}_{\mathbf{h}_{2}}\right) \geq \sum_{i=0}^{M R-1} \frac{1}{\left(c_{2} L \rho_{s}+c_{3}(L / R) \rho_{t}+c_{4}+\left(1 / \sigma_{h_{2}, i}^{2}\right)\right)},
$$

where the RHS is not related with $\mathbf{X}$ or $\mathbf{T}_{r}$ and can immediately serve as the lower bound of $\operatorname{tr}\left(\mathrm{BCRB}_{\mathbf{h}_{2}}\right)$. The lower bound is achieved when $\left(\mathbf{T}_{r}^{*} \otimes \mathbf{I}_{M}\right)\left(\mathbf{T}_{r}^{T} \otimes \mathbf{I}_{M}\right)=(L / R) \rho_{t} \mathbf{I}_{M R}$. Obviously, the choice $\left|\tilde{t}_{r, 0}\right|^{2}=\cdots=\left|\tilde{t}_{r, R L-1}\right|^{2}=\rho_{t} / R$ satisfies the requirement. (C2).

Combing both proofs yields the conditions of $(\mathrm{Cl})$ and

\section{Calculation of Correlations}

$R_{\mathrm{H}}$ is computed as

$$
\begin{aligned}
R_{\mathbf{H}} & =E\left\{\mathbf{H}^{H} \mathbf{H}\right\} \\
& =\frac{\alpha^{2} \rho_{r}}{R\left(1+\rho_{s}\right)} E\left\{\left(\mathbf{H}_{1}^{H} \mathbf{H}_{2}^{H}\right)\left(\mathbf{H}_{2} \mathbf{H}_{1}\right)\right\} \\
& =\frac{\alpha^{2} \rho_{r}}{R\left(1+\rho_{s}\right)} E\left\{\mathbf{H}_{1}^{H} E\left\{\mathbf{H}_{2}^{H} \mathbf{H}_{2}\right\} \mathbf{H}_{1}\right\} \\
& =\frac{\alpha^{2} \rho_{r}}{R\left(1+\rho_{s}\right)} E\left\{\mathbf{H}_{1}^{H} R_{\mathbf{H}_{2}} \mathbf{H}_{1}\right\},
\end{aligned}
$$

where $R_{\mathrm{H}_{2}}$ is given as

$$
\begin{aligned}
R_{\mathbf{H}_{2}} & =E\left\{\mathbf{H}_{2}^{H} \mathbf{H}_{2}\right\} \\
& =E\left\{\mathbf{H}_{w 2}^{H} R_{d}^{H / 2} R_{d}^{1 / 2} \mathbf{H}_{w 2}\right\} \\
& =E\left\{\mathbf{H}_{w 2}^{H} R_{d}^{H} \mathbf{H}_{w 2}\right\} \\
& =E\left\{\mathbf{H}_{w 2}^{H} \mathbf{U}_{d} \mathbf{D}_{d} \mathbf{U}_{d}^{H} \mathbf{H}_{w 2}\right\} \\
& =E\left\{\mathbf{H}_{w 2}^{H} \mathbf{D}_{d} \mathbf{H}_{w 2}\right\} \\
& =\operatorname{tr}\left(R_{d}\right) \mathbf{I}_{R} \\
& =M \mathbf{I}_{R} .
\end{aligned}
$$

Thus,

$$
\begin{aligned}
R_{\mathbf{H}} & =\frac{\alpha^{2} \rho_{r} M}{R\left(1+\rho_{s}\right)} E\left\{\mathbf{H}_{1}^{H} \mathbf{H}_{1}\right\} \\
& =\frac{\alpha^{2} \rho_{r} M}{R\left(1+\rho_{s}\right)} R_{s}^{H / 2} E\left\{\mathbf{H}_{w 1}^{H} \mathbf{H}_{w 1}\right\} R_{s}^{1 / 2} \\
& =\frac{\alpha^{2} \rho_{r} M}{\left(1+\rho_{s}\right)} R_{s} \\
& =\frac{\alpha^{2} \rho_{r} M}{\left(1+\rho_{s}\right)} \mathbf{I}_{N} .
\end{aligned}
$$

$R_{\mathrm{N}}$ is computed as

$$
\begin{aligned}
R_{\mathbf{N}} & =E\left\{\mathbf{N}^{H} \mathbf{N}\right\} \\
& =M \mathbf{I}_{L}+\frac{\alpha^{2} \rho_{r}}{R\left(1+\rho_{s}\right)} E\left\{\mathbf{W}_{R}^{H} R_{\mathbf{H}_{2}} \mathbf{W}_{R}\right\} \\
& =M \mathbf{I}_{L}+\frac{\alpha^{2} \rho_{r} M}{\left(1+\rho_{s}\right)} \mathbf{I}_{L} \\
& =\frac{M\left(1+\rho_{s}+\alpha^{2} \rho_{r}\right)}{\left(1+\rho_{s}\right)} \mathbf{I}_{L} .
\end{aligned}
$$

\section{Acknowledgment}

This research was supported by the National Science Foundation of China (Grant no. NFSC \# 61071083).

\section{References}

[1] B. Wang, J. Zhang, and A. Høst-Madsen, "On the capacity of MIMO relay channels," IEEE Transactions on Information Theory, vol. 51, no. 1, pp. 29-43, 2005.

[2] H. Bölcskei, R. U. Nabar, O. Oyman, and A. J. Paulraj, "Capacity scaling laws in MIMO relay networks," IEEE Transactions on Wireless Communications, vol. 5, no. 6, pp. 1433-1444, 2006.

[3] Y. Fan and J. Thompson, "MIMO configurations for relay channels: theory and practice," IEEE Transactions on Wireless Communications, vol. 6, no. 5, pp. 1774-1786, 2007.

[4] X. Tang and Y. Hua, "Optimal design of non-regenerative MIMO wireless relays," IEEE Transactions on Wireless Communications, vol. 6, no. 4, pp. 1398-1406, 2007.

[5] S. W. Peters and R. W. Heath, "Nonregenerative MIMO relaying with optimal transmit antenna selection," IEEE Signal Processing Letters, vol. 15, pp. 421-424, 2008.

[6] C. B. Chae, T. Tang, R. W. Heath, and S. Cho, "MIMO relaying with linear processing for multiuser transmission in fixed relay networks," IEEE Transactions on Signal Processing, vol. 56, no. 2, pp. 727-738, 2008.

[7] A. S. Behbahani, R. Merched, and A. M. Eltawil, "Optimizations of a MIMO relay network," IEEE Transactions on Signal Processing, vol. 56, no. 10, pp. 5062-5073, 2008.

[8] R. Zhang, C. C. Chai, and Y. C. Liang, "Joint beamforming and power control for multiantenna relay broadcast channel with QoS constraints," IEEE Transactions on Signal Processing, vol. 57, no. 2, pp. 726-737, 2009.

[9] C. S. Patel and G. L. Stüber, "Channel estimation for amplify and forward relay based cooperation diversity systems," IEEE Transactions on Wireless Communications, vol. 6, no. 6, pp. 2348-2355, 2007.

[10] F. Gao, T. Cui, and A. Nallanathan, "On channel estimation and optimal training design for amplify and forward relay networks," IEEE Transactions on Wireless Communications, vol. 7, no. 5, pp. 1907-1916, 2008.

[11] J. Pang, G. Shen, D. Wang, L. Jiang, and W. Wang, "Channel estimation and optimal training design for amplify and forward MIMO relay channel under spatial fading correlation," in Proceedings of the IEEE 72nd Vehicular Technology Conference Fall (VTC2010-Fall'10), pp. 1-5, September 2010. 
[12] J. Ma, P. Orlik, J. Zhang, and G. Y. Li, "Pilot matrix design for estimating cascaded channels in two-hop MIMO amplifyand-forward relay systems," IEEE Transactions on Wireless Communications, vol. 10, no. 6, pp. 1956-1965, 2011.

[13] O. Muñoz-Medina, J. Vidal, and A. Agustín, "Linear transceiver design in nonregenerative relays with channel state information," IEEE Transactions on Signal Processing, vol. 55, no. 6, pp. 2593-2604, 2007.

[14] I. Hammerström and A. Wittneben, "Power allocation schemes for amplify-and-forward MIMO-OFDM relay links," IEEE Transactions on Wireless Communications, vol. 6, no. 8, pp. 2798-2802, 2007.

[15] M. Herdin, "A chunk based ofdm amplify-and-forward relaying scheme for $4 \mathrm{~g}$ mobile radio systems," in Proceedings of the IEEE International Conference on Communications (ICC '06), vol. 10, pp. 4507-4512, June 2006.

[16] Y. Li, W. Wang, J. Kong, and M. Peng, "Subcarrier pairing for amplify-and-forward and decode-and-forward OFDM relay links," IEEE Communications Letters, vol. 13, no. 4, pp. 209211, 2009.

[17] T. Kong and Y. Hua, "Optimal channel estimation and training design for MIMO relays," in Proceedings of the 44th Asilomar Conference on Signals, Systems and Computers (ASILOMAR '10), pp. 663-667, November 2010.

[18] T. Kong and Y. Hua, "Optimal design of source and relay pilots for mimo relay channel estimation," IEEE Transactions on Signal Processing, vol. 59, no. 9, pp. 4438-4446, 2011.

[19] O. Muñoz-Medina, J. Vidal, and A. Agustín, "Linear transceiver design in nonregenerative relays with channel state information," IEEE Transactions on Signal Processing, vol. 55, no. 6, pp. 2593-2604, 2007.

[20] J. Ma, P. Orlik, J. Zhang, and G. Y. Li, "Pilot matrix design for interim channel estimation in two-hop MIMO AF relay systems," in Proceedings of the IEEE International Conference on Communications (ICC '09), June 2009.

[21] P. Lioliou and M. Viberg, "Least-squares based channel estimation for MIMO relays," in Proceedings of the International ITG Workshop on Smart Antennas (WSA '08), pp. 90-95, February 2008.

[22] F. Gao, B. Jiang, X. Gao, and X. -D. Zhang, "Superimposed training based channel estimation for ofdm modulated af relay networks," in Proceedings of the IEEE International Conference on Communications (ICC '06), pp. 1-5, June 2011.

[23] F. Gao, B. Jiang, X. Gao, and X. D. Zhang, "Superimposed training based channel estimation for OFDM modulated amplify-and-forward relay networks," IEEE Transactions on Communications, vol. 59, no. 7, pp. 2029-2039, 2011.

[24] D. S. Shiu, G. J. Foschini, M. J. Gans, and J. M. Kahn, "Fading correlation and its effect on the capacity of multielement antenna systems," IEEE Transactions on Communications, vol. 48, no. 3, pp. 502-513, 2000.

[25] C. N. Chuah, D. N. C. Tse, J. M. Kahn, and R. A. Valenzuela, "Capacity scaling in MIMO wireless systems under correlated fading," IEEE Transactions on Information Theory, vol. 48, no. 3, pp. 637-650, 2002. 

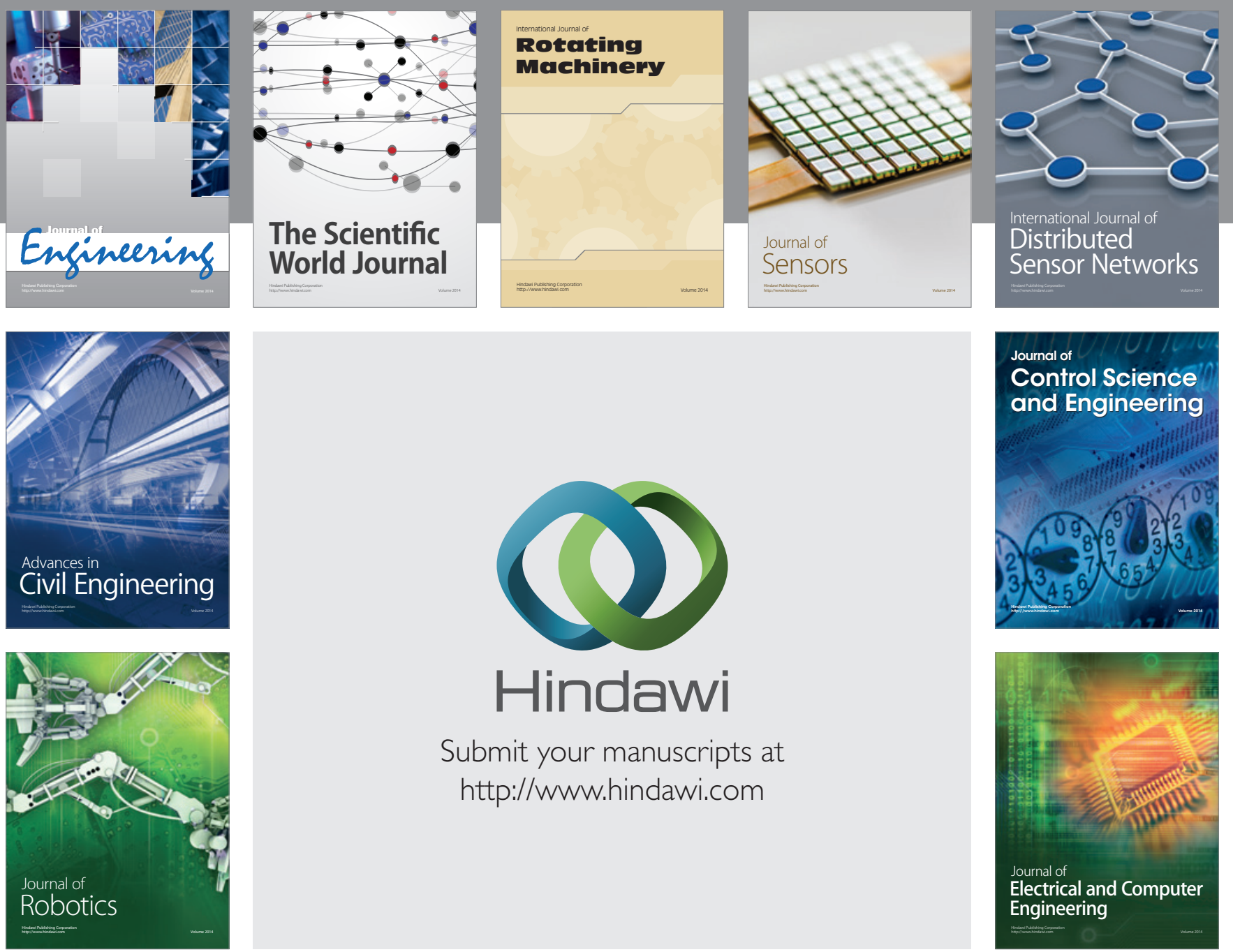

Submit your manuscripts at

http://www.hindawi.com
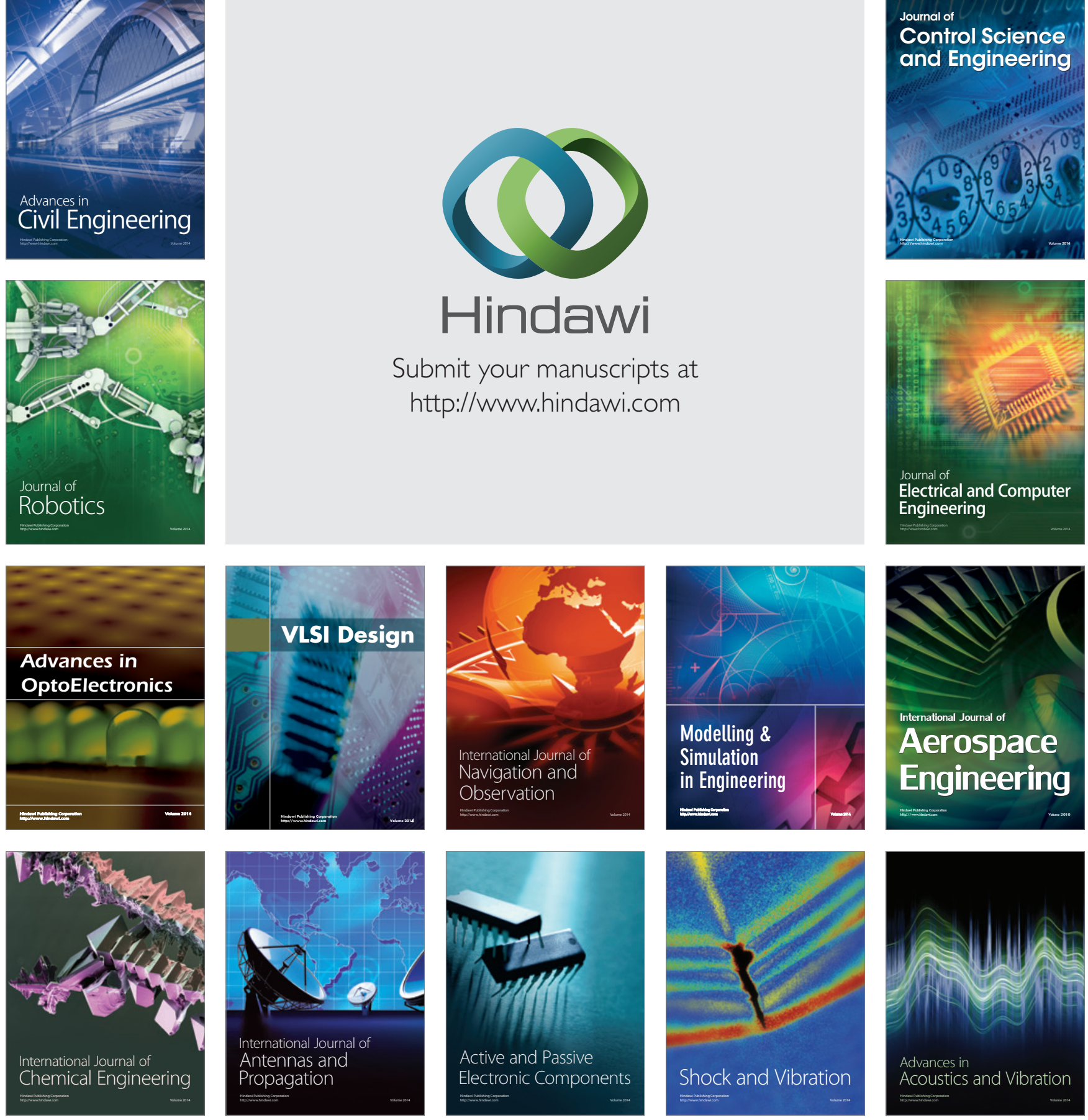